\title{
Power Doppler Ultrasonography on Tendons of Lower Limbs to Assess Subclinical Enthesopathy in Patients with Psoriasis: A Cross Sectional Study
}

Abdelazeem M. ElHefny', MaryamA. Abdel-Rahman ${ }^{1}$, Nermeen N. Aziz ${ }^{1}$, Nehal A. Hassanen $^{1}$

Internal medicine Department, Faculty of Medicine, Ain Shams University.

\begin{abstract}
Background: Psoriatic arthritis is a long-term condition that affects some people who have psoriasis. In severe cases, there's a risk of the joints becoming permanently damaged or deformed. Diagnosis of psoriasis-associated enthesopathy helps early detection and treatment of psoriatic arthropathy before joint damage becomes established.
\end{abstract}

Objective: to detect the presence of asymptomatic entheseal abnormalities in psoriatic patients in an attempt for preclinical detection of psoriatic arthritis before full joint affection becomes established.

Methods: A cross-sectional study included 50 with psoriasis without evidence of arthropathy collected from Ain Shams University hospitals. All the patients were subjected to the following: Full history, clinical examination, routine laboratory investigations and power doppler US on both lower limb entheses.

Results: The present study proved that 48 patients out of 50 with psoriasis showed entheseal abnormalities at least in one site of lower limbs detected by MSUS and this was assessed using glascow ultrasound enthesopathy scoring system (GUESS).

Conclusion: MSUS is useful in the detection of early findings of psoriatic arthropathy especially the presence of enthesitis even in asymptomatic patients.

Keywords: subclinical enthesopathy, psoriatic arthritis, musculoskeletal ultrasound. 


\section{Introduction}

Psoriasis is an inflammatory skin disease characterized by itchy, erythematous, scaly lesions, papules and sometimes pustules (Boehncke and Schön, 2015).(1)

About $11 \%$ of those diagnosed with psoriasis have also been diagnosed with PsA. However, about $30 \%$ of people with psoriasis will eventually develop PsA. While joint affection is the main distinguishing feature between PsA and psoriasis, a well known extra-articular feature of PsA is tendinopathy and enthesopathy (Ritchlin et al., 2017).(2)

MSUS has been known to be a reliable tool in detecting enthesitis and is more accurate than clinical examination, conventional radiography, and even MRI, especially during the early stages of inflammatory process in enthesitis (Ozçakar et al., 2005).(3)

Furthermore, occult enthesitis detected using MSUS, especially with a power Doppler signal, has a predictive value for the occurance of joint changes in patients with psoriasis (El Miedany et al., 2015).(4)

\section{Materials and Methods}

Through a cross sectional study; Fifty patients with psoriasis without evidence of arthropathy were collected randomly by consecutive method from Ain Shams university hospital. Inclusion criteria were Age $>18$ years and patients with only skin manifestations of psoriasis.

Every participant was subjected to the following:

(a) Detailed history taking and clinical examination.

(b) Measuring severity and extent of psoriasis using Psoriasis Area and Severity Index (PASI score) (Fredriksson and Pettersson, 1978).(5)

(c) Laboratory work including CBC, CRP and ESR.

(d) Musculoskeletal ultrasonography mode/grey scale (GS) assessment and power Doppler (PD) on both lower limbs' tendons to assess enthesopathy using glascow enthesitis scoring system (GUESS). 


\section{Statistical analysis}

The collected data were coded, tabulated, and statistically analyzed using IBM SPSS statistics (Statistical Package for Social Sciences) software version 22.0, IBM Corp., Chicago, USA, 2013. The level of significance was taken at $\mathrm{P}$ value $<0.05$ is significant, otherwise is non-significant.

\section{Results}

Table [1] shows the study participants (28 males and 22 females) with age ranges from 18 to 73 years (mean, $45.5 \pm 14.6$ years), the disease duration ranged from 0.8 to 50 years (mean, $9.4 \pm 9.9$ years) and BMI ranges from 28.5 to 46.8 (mean 28.5 \pm 6.4 ).

\section{Table (1): Characteristics of the Studied Population:}

\begin{tabular}{||l|lll||}
\hline Data & Mean & \pm & SD (range) \\
\hline Age (Years) & 45.580 & \pm & $14.686(18-73)$ \\
\hline Disease duration (Years) & 9.456 & \pm & $9.970(0.8-50)$ \\
\hline BMI & 28.536 & \pm & $6.422(18.3-46.8)$ \\
\hline Age of onset & 36.136 & \pm & $16.492(6-66)$ \\
\hline
\end{tabular}

Table [2] shows highly significant correlation between total GUESS, age of the patient and age of onset of the disease. otherwise, there isn't any significant difference concerning other parameters (PASI, disease duration, BMI and any laboratory investigations).

Table (2): Relationship between GUESS\& other parameters:

\begin{tabular}{|l|l|l|}
\hline \multirow{2}{*}{ Parameter } & \multicolumn{2}{|l|}{ Total GUESS } \\
\cline { 2 - 3 } & $\mathbf{r}$ & P-value \\
\hline Age (Years) & 0.546 & $<0.001^{*}$ \\
\hline Disease duration (Years) & 0.072 & 0.620 \\
\hline BMI & 0.207 & 0.149 \\
\hline Age of onset & 0.443 & $0.001^{*}$ \\
\hline PASI score & 0.031 & 0.828 \\
\hline
\end{tabular}




\section{Discussion}

Enthesitis is much more than local inflammation; indeed, it is considered as the primary pathological process underling SpA (Watad et al .,2018).(6)

The diagnosis of enthesitis in clinical practice is usually based on conventional radiography, which can only demonstrate established bony erosions and spurs and gives little information regarding soft tissues, particularly during the early phases of enthesopathy (Moshrif et al ., 2017).(7)

In recent years, ultrasonography has proved to be a highly sensitive and noninvasive tool, especially in the assessment of tendon and joint involvement (Hassan et al., 2014).(8)

In our study, we aimed to assess involvement of entheses in psoriatic patients without rheumatological complaints. We recorded and analysed some demographic and clinical data that could have an effect on the presence of power doppler ultrasound abnormalities.

Our results proved that 48 patients out of 50 with psoriasis showed entheseal abnormalities at least in one site of lower limbs detected by MSUS and this was assessed using glascow ultrasound enthesopathy scoring system (GUESS).

Naredo et al., 2011(9) reported that 162 patients with plaque psoriasis without musculoskeletal complaints were found to have a significant prevalence of enthesopathy detected by US (62.5\%) also, Elnady et al.,2019(10) who showed that MSUS subclinical enthesitis is a relatively common finding in psoriasis patients free from musculoskeletal manifestations.

Hassan et al., in 2014(8) found that the number of abnormal entheses by clinical examination in early spondyloarthritis patients was 52 per $360(14 \%)$, whereas the number found by ultrasonographic examination was 239 per 360 (66.3\%). This shows that sonography is very important to assess enthesis better than clinical examination.

On the contrary, Freeston et al., 2012(11) have shown that the prevalence of subclinical active enthesitis in early PsA is low. The small number of subclinically inflamed entheses were found in the lower extremities.

In our study, a significant correlation between age and enthesopathy detected by MSUS. No correlation could be established between sex, disease duration, BMI, smoking, diabetes mellitus, nail involvement, mechanical stress, severity of the disease represented by PASI score and preclinical enthesitis represented by GUESS score.

Our results agree with the study of Moshrif et al., in 2017(7) who found a significant correlation between the patient's age and US enthesitis and no significant difference 
between the sex distribution and US abnormalities. On the contrary, he found a significant correlation between the disease duration and US enthesitis.

El Nady et al ., 2019(10) published that psoriatic patients without MSUS manifestations did not differ statistically regarding sex, age, duration of psoriasis, and PASI score. Furthermore, the area affected by psoriasis whether the scalp, nail, or skin only did not affect the incidence of MSUS manifestations and this agrees with our results.

Takata et al., in 2016(12) described that PsA is well associated with scalp, nail, or interglutel/buttock psoriasis. These distributions of psoriasis tended to be more frequent among subclinical PsA patients and this is against our results which showed that there is no significant correlation between PASI, distribution of psoriasis and entheseal abnormalities.

These contradictory results could be due to different inclusion criteria, different ethencity in different studies and also due to smallnumber of patients included in our study.

\section{Conclusion}

Enthesitis is an early manifestation of psoriatic arthritis. From our study we can conclude that MSUS is useful in the detection of early findings of psoriatic arthropathy especially the presence of enthesitis even in asymptomatic patients. Diagnosis of psoriasis-associated enthesopathy helps early detection and treatment of psoriatic arthropathy before joint damage becomes established.

\section{Ethical considerations}

The study followed the ethical considerations according to the ethical committee of Ain Shams, faculty of medicine. An informed written consent was taken from all patients participating in the study.

\section{Disclosure Statement}

There is no conflict of interest.

\section{References:}

1. Boehncke WH and Schön MP: Psoriasis. Lancet 2015;(386):983-994 
2. Ritchlin CT, Colbert RA and Gladman DD: Psoriatic arthritis. N Engl J Med.2017;(376):957-970

3. Ozçakar L, Cetin A, Inanici F, Kaymak B, Gurer CK., et al: Ultrasono-graphical evaluation of the Achilles tendon in psoriasis patients. Int J Dermatol. 2005;(44):930932

4. El Miedany Y, El Gaafary M, Youssef S, Ahmed I and Nasr A: Tailored approach to early psoriatic arthritis patients: clinical and ultrasono-graphic predictors for structural joint damage. Clin Rheumatol. 2015; (34):307-313

5. Fredriksson T, Pettersson U (1978): Severe psoriasis- Oral therapy with a new retinoid. Dermatologica 178;157:238-244.

6. Watad A, Cuthbert RJ, Amital H, McGonagle D. Enthesitis: Much More Than Focal Insertion Point Inflammation. Curr Rheumatol Rep. 2018 ;20(7):41.

7. Moshrif A, Mosallam A, Mohamed EE, Gouda W and Doma M: Subclinical enthesopathy in patients with psoriasis and its association with other disease parameters: a power Doppler ultrasonographic study. Eur J Rheumatol. 2017;(1):2428

8. Hassan A, Darwish A, Mohamed F, Ibrahim M and El-Karima A: Value of musculoskeletal ultrasonography in the diagnosis of peripheral enthesopathy in early spondyloarthropathy. Egyptian rheumatology and rehabilitation 2014 ;(41):51-57

9- Naredo E, Möller I, de Miguel E, Batlle-Gualda E, Acebes $\mathbf{C}$ et al ., High prevalence of ultrasonographic synovitis and enthesopathy in patientswith psoriasis without psoriatic arthritis: a prospective case-control study.Rheumatology (Oxford). 2011;(10):1838-1848

10. Elnady B, El Shaarawy NK, Dawoud NM, Elkhouly T, Desouky DE., et al : Subclinical synovitis and enthesitis in psoriasis patients and controls by ultrasonography in Saudi Arabia; incidence of psoriatic arthritis during two years. Clin Rheumatol. $2019 ;(6): 1627-1635$. 
11- Freeston JE, Coates LC, Helliwell PS, Hensor EM, Wakefield RJ., et : Is there subclinical enthesitis in early psoriatic arthritis? A clinical comparison with power doppler ultrasound. Arthritis Care Res (Hoboken). 2012 ;(10):1617-1621

12. Takata T, Takahashi A, Taniguchi Y, Terada Y and Sano S: Detection of asymptomatic enthesitis in psoriasis patients: An onset of psoriatic arthritis? J Dermatol. 2016 Jun;43(6):650-4. 